\title{
Photoinduced Chern insulating states in semi-Dirac materials
}

\author{
Kush Saha \\ Department of Physics and Astronomy, University of California, Irvine, California 92697, USA \\ California Institute for Quantum Emulation, Santa Barbara, California 93106, USA
}

(Dated: November 9, 2018)

\begin{abstract}
Two-dimensional (2D) semi-Dirac materials are characterized by a quadratic dispersion in one direction and a linear dispersion along the orthogonal direction. We study the topological phase transition in such $2 \mathrm{D}$ systems in the presence of an electromagnetic field. We show that a Chern insulating state emerges in a semi-Dirac system with two gapless Dirac nodes in the presence of light. In particular, we show that the intensity of a circularly polarized light can be used as a knob to generate topological states with nonzero Chern number. In addition, for fixed intensity and frequency of the light, a semi-Dirac system with two gapped Dirac nodes with trivial band topology can reveal the topological transition as a function of polarization of the light.
\end{abstract}

Introduction- Recent years have witnessed unprecedented theoretical and experimental advances in the field of materials whose low-energy excitations are described by Dirac fermions [1 $[-6]$. Such materials with a bulk gapped spectrum (e.g., 2D $\mathrm{HgTe} / \mathrm{CdTe}$ quantum

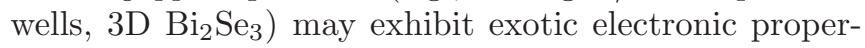
ties such as a quantum spin Hall effect 7], topological magneto-caloric effect [8], etc. Likewise, gapless or semimetallic Dirac materials (e.g., 2D graphene, 3D Weyl metal) may reveal many unconventional properties such as a quantum Hall effect at room temperature (particularly in graphene) 9], chiral anomaly induced negative magnetoresistance 10] (particularly in a Weyl metal), and many more 2, 11]. Due to their exotic properties, on the one hand, there has been a considerable effort to search for new materials with Dirac-like properties. This has led to several proposals to realize a new class of materials with specific 2D Dirac dispersion: parabolic in one direction and linear in the perpendicular direction. The materials or systems that can host such semi-Dirac (SD) dispersion are $\mathrm{TiO}_{2} / \mathrm{V}_{2} \mathrm{O}_{3} 12$, BEDT- $\mathrm{TTF}_{2} \mathrm{I}_{3}$ salt under pressure 13., hexagonal lattices in the presence of a magnetic field [14], and photonic system 15]. Due to their unusual dispersion, they may exhibit exotic properties [16, 17] in contrast to graphene or conventional $2 \mathrm{D}$ systems.

On the other hand, there is a race to engineer new materials with topological properties due to the lack of natural materials with such properties. The static controllable parameters by which one can induce topological phases in intrinsically nontopological materials include pressure 18], doping [19], disorder 20], temperature 21], and a few more [22]. However, most of these tools lack a continuous and high degree of controllability. Recently, it has been shown and later verified by experiment that time-dependent perturbations can induce topological phases in an intrinsically nontopological insulating material [23 36]. Also, it has been shown that polarized light can open a gap in semi-metallic systems, allowing them to host topologically protected gapless modes responsible for robust transport properties. Indeed, cir-
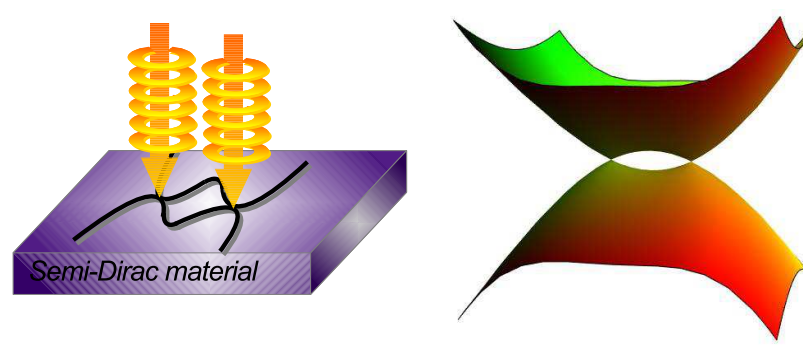

FIG. 1. a) Schematics of shining polarized light on the surface of a SD material. The size of the sample is considered to be smaller than the wavelength of the light. b) Energy spectrum of the SD Hamiltonian in Eq. (1) with two nodal points at $\left( \pm \sqrt{\delta_{0} / \alpha}, 0\right)$. Note that, this dispersion is gapped if $\delta_{0}<0$.

cularly polarized light opens a gap in a gapless Dirac system such as graphene, and may give rise to a photoinduced Hall current without applying any magnetic field 23]. Thus semi-metallic graphene becomes a Chern insulator (CI) in the presence of electromagnetic radiation, making it potential candidate for spintronics or transistors.

This raises an interesting question if semi-Dirac systems can carry a nonzero Chern number in the presence of polarized light. In this article, we have answered this affirmatively. For circularly polarized light, we show light can open a gap and induce topological states in a semimetallic SD system. In particular, the strength of the polarized light can be used as a knob to drive a topological transition. In addition, we show that a SD system with a trivial insulating phase can mimic the Haldane model as a function of polarization of the light. Finally, we investigate photoinduced anomalous Hall conductivity for different strengths of the incident beam. Note that a similar system was studied [37] recently in the presence of laser light but overlooked the interesting topological transition as presented here.

Model- The minimal model Hamiltonian describing low-energy electronic bands of a two-dimensional semi- 


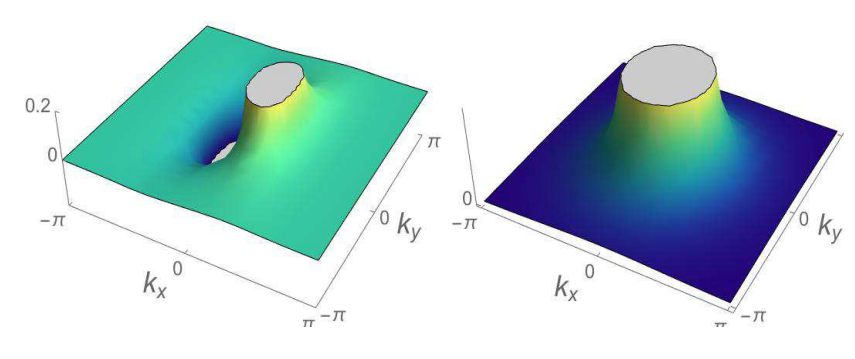

FIG. 2. Berry curvature of a gapped semi-Dirac Hamiltonian in the presence of perturbation $\delta H=m_{z} \sigma_{z}$. The integral of the Berry curvature over the BZ turns out to be zero since $\Omega\left(-k_{x},-k_{y}\right)=-\Omega\left(k_{x}, k_{y}\right)$ as a manifestation of time-reversal symmetry. b) Same plot as (a) for 2D linear Dirac dispersion [38]. In this case, the perturbation breaks time-reversal symmetry and leads to a nonzero Chern number [38], as is evident from the Berry curvature.

Dirac material is 16,17

$$
H_{0}(\mathbf{k})=\mathbf{d}(\mathbf{k}) \cdot \sigma
$$

where $\sigma=\left(\sigma_{x}, \sigma_{y}, \sigma_{z}\right)$ are the Pauli matrices in pseudospin space, $\mathbf{d}(\mathbf{k})=\left(\alpha k_{x}^{2}-\delta_{0}, v k_{y}, 0\right)$, where $\mathbf{k}=$ $\left(k_{x}, k_{y}\right)$ is the crystal momentum, $\alpha$ is the inverse of quasiparticle mass along $x, v$ is the Dirac velocity along $y$, and $\delta_{0}$ is the gap parameter. The energy eigenvalues are given by

$$
E_{k_{x}, k_{y}}^{ \pm}= \pm \sqrt{\left(\alpha k_{x}^{2}-\delta_{0}\right)^{2}+v^{2} k_{y}^{2}}
$$

where \pm denote the conduction and valence band, respectively. For $\delta_{0}=0$, the spectrum is gapless with linear dispersion along $k_{y}$. For $\delta_{0}<0$, it is a gapped system with trivial insulating phase. For $\delta_{0}>0$, we obtain two gapless Dirac points at $\left( \pm \sqrt{\delta_{0} / \alpha}, 0\right)$. The corresponding energy spectrum is shown in Fig. (1)b. Note that, this model obeys effective time-reversal $(\Theta=\mathcal{K})$, particlehole $\left(\mathcal{P}=\sigma_{y}\right)$ and chiral symmetries $\left(\mathcal{C}=\sigma_{y} \mathcal{K}\right)$, where $\mathcal{K}$ is the complex conjugation operator. It is worth mentioning that, in typical SD systems, the gapless modes are protected by mirror symmetry along some symmetry line [39] and cannot be destroyed without breaking that symmetry.

Berry curvature and Chern number- Eq. (11) represents a generic Hamiltonian of a two-level system. Its simple form in terms of "vector field" $\mathbf{d}(\mathbf{k})$ allows us to write the Berry curvature as

$$
\boldsymbol{\Omega}(\mathbf{k})=\frac{1}{2} \frac{\mathbf{d}(\mathbf{k})}{|\mathbf{d}(\mathbf{k})|^{3}}
$$

Since the Chern number is defined as an integral of Berry curvature over the $2 \mathrm{D}$ Brillouin zone (BZ), it can be expressed as

$$
C=\int d \mathbf{k} \Omega(\mathbf{k})=\frac{1}{2 \pi} \int d \mathbf{k} \frac{1}{2|\mathbf{d}|^{3}} \mathbf{d} \cdot\left(\partial_{k_{x}} \mathbf{d} \times \partial_{k_{y}} \mathbf{d}\right),
$$

where $\partial_{x}$ is the partial derivative with respect to $x$. Since the $z$-component of $\mathbf{d}$ in Eq. (1) is zero, the Berry curvature is zero everywhere except at the gapless Dirac points where it diverges. The perturbation $\delta H=m_{z} \sigma_{z}$ opens a gap and leads to a nonzero local Berry curvature,

$$
\Omega\left(k_{x}, k_{y}\right)= \pm \frac{2 \alpha v m_{z} k_{x}}{\left(E_{k_{x}, k_{y}}\right)^{3}} .
$$

Note that $\Omega\left(k_{x}, k_{y}\right)$ is asymmetric in $k_{x}$ for constant $m_{z}$. Thus, the Chern number $C$ is obtained to be zero, as also apparent from the Berry curvature shown in Fig. (2)a. The above perturbation therefore cannot change the band topology in the SD system. This is in contrast to half semimetals with 2D linear Dirac bands near the Fermi level, where a mass gap induced by spinorbit coupling leads to a nonzero Chern number [38, 40]. It is worth pointing out that, in graphene, the perturbation $m_{z} \sigma_{z}$, namely the "Semenoff mass" leads to a trivial insulating phase, while perturbation $m_{z} \sigma_{z} \tau_{z}$, namely the "Haldane mass" gives rise to non-trivial band topology, where $\tau_{z}$ denotes valley the degrees of freedom [41].

Floquet theory- We now investigate the effect of timedependent radiation generated by a radio frequency source or a laser on the semi-metallic phase of the SD Hamiltonian in Eq. (11). The light field $\mathbf{A}(t)=$ $A_{0}(\sin (\omega t), \sin (\omega t+\phi))$ minimally couples to the system via the momentum $\mathbf{k} \rightarrow \mathbf{k}+e \mathbf{A}(t)$, where $\phi$ is the polarization of the light, $e$ is the electric charge, $\omega$ is the frequency of the light, and $A_{0}$ is the strength of the applied field. Note that we neglect spatial dependence of the light field considering the fact that the wavelength of the light field is large compared to the sample size.

Then, in the presence of a light field, Eq. (11) can be read off as

$$
H(\mathbf{k}, t)=H_{0}(\mathbf{k})+H_{1}(\mathbf{k}, t) \sigma_{x}+H_{2}(\mathbf{k}, t) \sigma_{y},
$$

where $H_{1}(\mathbf{k}, t)=\frac{\alpha e^{2} A_{0}^{2}}{2}(1-\cos (2 \omega t))+2 \alpha e A_{0} k_{x} \sin (\omega t)$, and $H_{2}(\mathbf{k}, t)=v e A_{0}^{2} \sin (\omega t+\phi)$.

Under this time-dependent Hamiltonian, the quantum state evolves as $\Psi(t)=U\left(t, t_{0}\right) \Psi\left(t_{0}\right)$, where $U\left(t, t_{0}\right)=$ $\tau \exp \left(-\frac{i}{\hbar} \int_{t_{0}}^{t} d t^{\prime} H\left(\mathbf{k}, t^{\prime}\right)\right)$, where $\tau$ is the time-ordering operator, and $t_{0}$ is the initial time of the perturbation. Using the Floquet formalism, we define an effective static Hamiltonian, namely Floquet Hamiltonian after a full time period $T$ as

$$
H_{F}(\mathbf{k})=\frac{i}{\hbar T} \ln (U(T))
$$

where we have assumed $t_{0}=0$. Following Ref. [42], we obtain the Floquet Hamiltonian up to first order in inverse frequency,

$$
H_{F}(\mathbf{k}) \simeq H_{F}^{0}+H_{F}^{1},
$$


where $H_{F}^{0}$ is obtained by time-averaging of $H(\mathbf{k}, t)$ :

$$
H_{F}^{0}=\frac{1}{T} \int_{0}^{T} H(\mathbf{k}, t)=H_{0}(\mathbf{k})+\frac{\alpha e^{2} A_{0}^{2}}{2} \sigma_{x},
$$

and

$$
\begin{aligned}
H_{F}^{1} & =\frac{1}{2 T i \hbar} \int_{0}^{T} d t_{1} \int_{0}^{t_{1}}\left[H\left(\mathbf{k}, t_{1}\right), H\left(\mathbf{k}, t_{2}\right)\right] d t_{2} \\
& =\left(m_{0}+\beta k_{x}^{2}+\gamma k_{x}+\eta k_{x} k_{y}\right) \sigma_{z},
\end{aligned}
$$

where $m_{0}=\frac{e A_{0} v}{\hbar \omega}\left(\alpha e^{2} A_{0}^{2}-2 \delta_{0}\right) \cos (\phi), \quad \beta=$ $\frac{e A_{0} v}{\hbar \omega} 2 \alpha \cos (\phi), \gamma=\frac{e^{2} A_{0}^{2} v}{\hbar \omega} 2 \alpha \sin (\phi)$ and $\eta=-\frac{4 \alpha e A_{0} v}{\hbar \omega}$. Note that this is a high-frequency expansion when only one photon is considered for the process. We recognize $H_{F}^{1}$ as a gap-opening mass term in Eq. (11). As already discussed, $H_{0}(\mathbf{k})$ represents the Hamiltonian of a trivial insulator in the presence of a gap induced by a constant mass term. However, we will see that the momentum dependent term in $H_{F}^{1}$ plays a crucial role in revealing the band topology in these systems.

Combining $H_{F}^{0}$ and $H_{F}^{1}$, Eq. (8) yields

$$
H_{F}(\mathbf{k})=\mathbf{n}(\mathbf{k}) \cdot \sigma,
$$

with

$$
\mathbf{n}(\mathbf{k})=\left(\alpha k_{x}^{2}+\delta_{1}, v k_{y}, m_{\mathrm{eff}}\right),
$$

where $\delta_{1}=\frac{\alpha e^{2} A_{0}^{2}}{2}-\delta_{0}, m_{\mathrm{eff}}=m_{0}+\beta k_{x}^{2}+\gamma k_{x}+\eta k_{x} k_{y}$. Note that the incident beam shifts the position of the Dirac nodes. Notice also, that $m_{\text {eff }}$ breaks the effective time-reversal symmetry as $\Theta m_{\mathrm{eff}}\left(k_{x}, k_{y}\right) \Theta^{-1} \neq$ $m_{\text {eff }}\left(-k_{x},-k_{y}\right)$.

With the new vector field $\mathbf{n}(\mathbf{k})$, we define the Chern number for the Floquet Hamiltonian as

$$
C_{\mathrm{F}}=\frac{1}{2 \pi} \int d \mathbf{k} \frac{1}{2|\mathbf{n}(\mathbf{k})|^{3}} \mathbf{n}(\mathbf{k}) \cdot\left(\partial_{k_{x}} \mathbf{n}(\mathbf{k}) \times \partial_{k_{y}} \mathbf{n}(\mathbf{k})\right),
$$

where $|n(\mathbf{k})|= \pm \sqrt{\left(\alpha k_{x}^{2}+\delta_{1}\right)^{2}+v^{2} k_{y}^{2}+m_{\text {eff }}^{2}}$.

Chern insulating states in gapless SD systems- Together with Eq. (12) and the integral part of Eq. (13), we obtain

$$
\Omega\left(k_{x}, k_{y}\right)=\frac{v}{2|n(\mathbf{k})|^{3}}\left[\gamma\left(\alpha k_{x}^{2}-\delta_{1}\right)-\eta k_{y}\left(\alpha k_{x}^{2}+\delta_{1}\right)\right] .
$$

It is apparent that $\Omega\left(k_{x}, k_{y}\right) \neq-\Omega\left(-k_{x},-k_{y}\right)$ as a consequence of broken time-reversal symmetry. We would like to point out that the numerator of $\Omega\left(k_{x}, k_{y}\right)$ does not contain any term involving $m_{0}$. However, the presence of such a term in the numerator can have important consequences in revealing the band topology as will be evident below.

For circularly polarized $(\phi= \pm \pi / 2)$ light, and for $\delta_{0}>\alpha e^{2} A_{0}^{2} / 2$, the gapless Dirac nodes become gapped
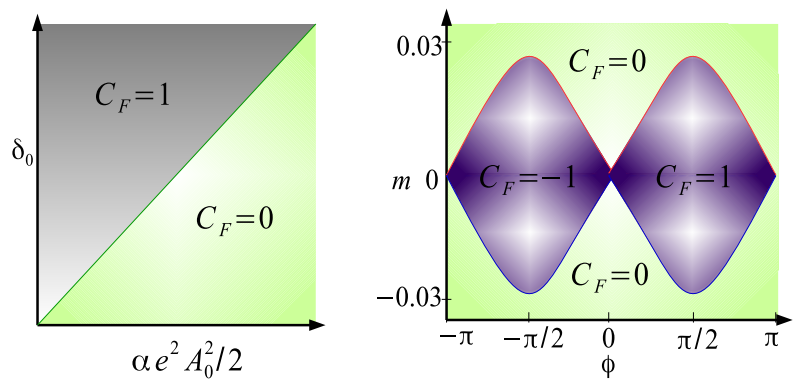

FIG. 3. a) Schematic phase diagram of the photoinduced Chern number $\left(C_{F}\right)$ in the $\delta_{0}-\alpha e^{2} A_{0}^{2} / 2$ plane. The diagonal lines correspond to $\delta_{0}=\alpha e^{2} A_{0}^{2} / 2$, where $C_{F}$ is ill-defined as the spectrum is gapless. b) Phase diagram of $C_{F}$ in the $m-\phi$ plane for a gapped semi-Dirac system as discussed in the text. The red $\left(m_{c_{1}}>0\right)$ and blue lines $\left(m_{c_{2}}<0\right)$ correspond to a gapless spectrum due to the gap closing at only one Dirac point.

with their new position at $\left( \pm \sqrt{\frac{\left|\delta_{1}\right|}{\alpha}-\frac{\gamma^{2}}{2 \alpha^{2}}}, 0\right)$. Then the total Berry flux for this gapped spectrum is found to be $\pm 2 \pi \operatorname{sgn}(\gamma)$, where \pm correspond to the positive and negative energy spectrum, respectively. Note that left $(\phi=-\pi / 2)$ or right circularly $(\phi=+\pi / 2)$ polarized light determines the sign of $\gamma$. The $2 \pi$ flux comes from the fact that the energy spectrum has two Dirac-like dispersions, and each of those contributes $\pm \pi$ to the total flux in contrast to $2 \pi$ flux in bilayer graphene due to quadratic band touching. Consequently, two gapped Dirac nodes make a contribution of $\pm(1 / 2+1 / 2)= \pm 1$ to $C_{F}$. The total $C_{F}$ is obtained by summing over the contribution coming from all Dirac nodes in the BZ. For typical semi-Dirac materials, there are four symmetryrelated momenta [14], which, in turn, give rise to a total of 8 Dirac nodes, hence a total $C_{F}= \pm 4$. However, for simplicity, we restrict ourselves to a system with only two Dirac nodes within the BZ 43].

For $\delta_{0}=\alpha e^{2} A_{0}^{2} / 2$, i.e., $\delta_{1}=0$, the spectrum is gapless. For $\delta_{0}<\alpha e^{2} A_{0}^{2} / 2$, the spectrum becomes gapped again. In this case, $C_{F}$ turns out to be zero. Thus varying intensity of the incident light, we can drive topological transition in a gapless SD system (cf. Fig. 33). Note that, unlike the case of gapped trivial insulating phase induced by constant mass term (as discussed earlier), the Berry curvature $\Omega\left(k_{x}, k_{y}\right)$ is not asymmetric either in $k_{x}$ or $k_{y}$. We therefore expect to have finite anomalous Hall conductivity at finite doping $\left(\epsilon_{F} \neq 0\right)$. This will be discussed shortly.

Chern insulating states in gapped SD systems- The objective of this section is to explore topological phenomena in a SD system with two gapped Dirac nodes within the BZ. The low-energy model Hamiltonian is given by

$$
H_{\mathrm{g}}(\mathbf{k})=H_{0}(\mathbf{k})+m \sigma_{z}
$$

As mentioned earlier, this Hamiltonian represents a triv- 
ial insulator with zero Chern number as a consequence of an asymmetric Berry curvature. The presence of polarized light leads to $n_{\mathrm{g}}(\mathbf{k})=\left(\alpha k_{x}^{2}+\delta_{1}-m \xi, v k_{y}+\right.$ $\left.m \chi k_{x}, m+m_{\text {eff }}\right)$, where $\xi=2 e A_{0} v \cos (\phi) / \hbar \omega$ and $\chi=$ $4 e A_{0} \alpha / \hbar \omega$. Note that the position of the Dirac nodes moves along both $k_{x}$ and $k_{y}$ directions as opposed to the case discussed in the preceding section. Note also, that the movement depends on the polarization of the light.

With this $n_{\mathrm{g}}(\mathbf{k})$, the numerator of the Berry curvature turns out to be dependent on $m$, and also on $\cos (\phi)$ [46], in contrast to the previous case where the term involving $m_{0}$ vanishes identically in the numerator. Consequently, for fixed intensity and frequency of the light, we can mimic the phase diagram of the Haldane model by varying $m$ with the polarization of the light. The corresponding plot is shown in Fig. (3) b. Along the curve lines $\left(m_{c_{1}} \simeq\right.$ $m_{c_{2}}=m_{c}$ ), one Dirac node closes while the other remains open, hence the spectrum becomes gapless. Note that $m_{c}$ depends on the intensity, frequency, and polarization of the light. For circularly polarized light, and for $\delta_{1}<0$, we obtain $m_{c}(\phi=\pi / 2)= \pm 2 e^{2} A_{0}^{2} v \hbar \omega \sqrt{\alpha\left|\delta_{1}\right|} /\left(16 e^{2} A_{0}^{2} \alpha \delta_{1}-\right.$ $\left.\hbar^{2} \omega^{2}\right)$. For any other polarization, $m_{c}$ can be obtained numerically for fixed $A_{0}$ and $\omega$. When $|m|<\left|m_{c}\right|$, the mass gaps at two Dirac nodes are opposite in sign, hence $C_{F}= \pm \operatorname{sgn}(\phi)$. In contrast, for $|m|>\left|m_{c}\right|$, the mass gaps at two Dirac nodes turn out to be the same in sign. Thus we obtain $C_{F}=0$, indicating a transition from a topological to a trivial phase as a function of polarization of the light.

Before ending this section, we would like to point out that a gapped SD system for $\delta_{0}<0$ in Eq. (1) does not host any interesting topological states in the presence of light. Thus the presence of gapless or gapped Dirac nodes (when $\delta_{0}>0$ ) in the field-free Hamiltonian of SD materials plays a crucial role in revealing the topological transition in the presence of light. In sum, this reflects a non-trivial interplay between light and band spectrum. Finally, we comment on the lattice model which is crucial to compute the Chern number correctly. In doing so, we consider the lattice Hamiltonian, $H=\left(1-2 \cos \left(k_{x}\right)\right) \sigma_{x}+$ $k_{y} \cos \left(k_{x}\right) \sigma_{y}$, which has two Dirac nodes within the $2 \mathrm{D}$ BZ. Note that, this model Hamiltonian can be obtained from hexagonal lattice geometry with appropriate band parameters [45]. Then the perturbation $\delta H=\gamma \sin \left(k_{x}\right) \sigma_{z}$ reveals the same topological properties as discussed in the preceding sections.

Anomalous Hall conductivity- Unlike quantum Hall conductivity, the anomalous Hall conductivity does not require any external magnetic field or nonzero Chern number. The local Berry curvature can contribute to conductivity even at zero temperature for finite Fermi energy, $\epsilon_{F}$. The Hall conductivity is defined as

$$
\sigma_{x y}^{ \pm}= \pm \frac{e^{2}}{\hbar} \int \frac{d \mathbf{k}}{(2 \pi)^{2}} f\left(E_{k_{x}, k_{y}}^{ \pm}\right) \Omega\left(k_{x}, k_{y}\right),
$$

where $f(\epsilon)$ is the Fermi-Dirac (FD) distribution func-
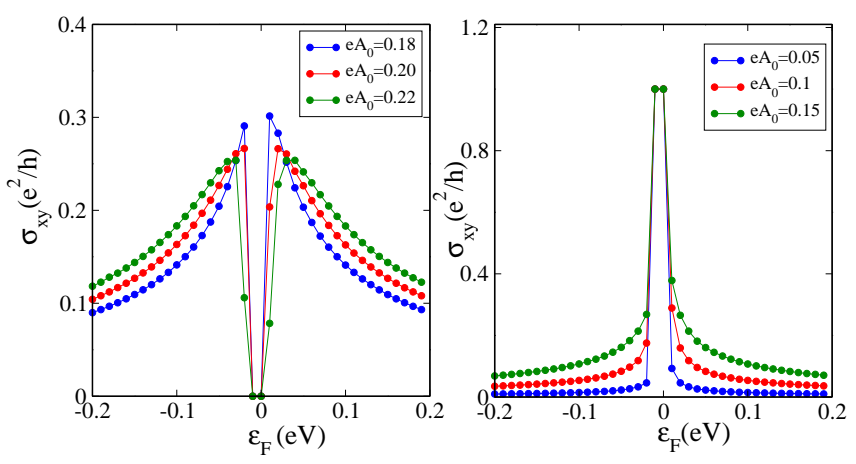

FIG. 4. a) Anomalous Hall conductivity $\sigma_{x y}$ for a trivial insulating phase with nonzero Berry curvature as a function of $\epsilon_{F}$. It is evident that $\sigma_{x y}$ increases with the intensity $A_{0}$. However, as soon as $\epsilon_{F}$ reaches the gap, it drops to zero as a consequence of zero Chern number for $\delta_{1}>0$. b) For $\delta_{1}<0$, we see the conductivity is quantized in units of $e^{2} / h$ if $\epsilon_{F}$ lies within the gap. Away from the gap, we obtain a very small contribution to conductivity since $\gamma$ is small for weak intensity $A_{0}$.

tion, $f(\epsilon)=\left(1+e^{\left(\epsilon-\epsilon_{F}\right) / K_{B} T}\right)^{-1}$, where $T$ is the temperature. The total conductivity is the sum of two $\sigma_{x y}^{ \pm} \mathrm{s}$. Note that Eq. (16) is best valid for equilibrium dc Hall conductivity. In nonequilibrium, the expression for $\sigma_{x y}$ differs due to the nonuniversal nature of the FD distribution, which, in turn, depends on the details of the systems, its environment, etc [23, 47, 48]. However, we approximate $\sigma_{x y}$ by its equilibrium value based on the fact that the energy of the perturbing field exceeds any other energy scales of the system 48]. At zero temperature, $f\left(E_{k_{x}, k_{y}}^{ \pm}\right)=\Theta\left(E_{k_{x}, k_{y}}^{ \pm}-\epsilon_{F}\right)$. For a typical semiDirac material, we take $v=0.65 \mathrm{eV} \stackrel{\circ}{A}, \alpha=0.75 \mathrm{eV} \AA^{2}$, $\delta_{0}=0.01 \mathrm{eV}$. The typical phonon energy that is used in experiment is roughly $0.25 \mathrm{eV}$ with $e A_{0}=0.01-0.2 \AA^{-1}$. Using these parameters, we compute the anomalous conductivity, particularly for a gapless SD system when $\delta_{0}<\alpha e^{2} A_{0}^{2} / 2$. Fig. (4) a shows the dependence of $\sigma_{x y}$ on $\epsilon_{F}$ and $A_{0}$. Note that $\epsilon_{F}=0$ corresponds to Fermi energy in the middle of the gap. As $\epsilon_{F}$ approaches to the gap, the conductivity gradually increases, and drops to zero as $\epsilon_{F}$ reaches the gap. Notice that, a measurable conductivity contribution can be obtained if $\epsilon_{F}$ lies close to the gap. Also, the magnitude of Hall conductivity varies with the strength of the vector potential provided that ve $A_{0}<<\hbar \omega$ as a valid criterion for Floquet expansion. Fig. (4) b shows zero temperature conductivity for $\delta_{0}>\alpha e^{2} A_{0}^{2} / 2$. As expected, the conductivity is quantized if $\epsilon_{F}$ lies within the gap. However, away from the gap the conductivity appears to be small. This is because of the low intensity set by the condition $\delta_{0}>\alpha e^{2} A_{0}^{2} / 2$.

The predicted photoinduced chiral nature of the $2 \mathrm{D}$ semi-Dirac materials can be verified by dynamical Hall measurement using a crossed ac electric and magnetic field of circularly polarized light [49]. In addition, since 
the intensity of the electromagnetic radiation is easily controllable in experiment, the signature of transition from a topological to a trivial phase can be tested in this transport experiment, provided that $\delta_{0}$ in the band spectrum is positive.

Conclusion- We have shown that light can induce topological states in an intrinsically trivial semi-Dirac materials. For circularly polarized light, the intensity of the light can be used to induce topological phases in a semi-metallic semi-Dirac system. On the other hand, for fixed intensity and frequency, the polarization of light can drive a topological transition in a gapped semi-Dirac system. In addition, the intensity of light can be used to tune the anomalous Hall conductivity to measurable values for Fermi energy lying either in the conduction or valence bands.

KS thanks M. Kolodrubetz, S. A. Parameswaran and I. Garate for useful discussions. This work is funded by NSF Grant DMR-1455366 and the California Institute for Quantum Emulation, supported by a President's Research Catalyst Award (CA-15-327861) from the University of California Office of the President.

[1] K. S. Novoselov, A. K. Geim, S. V. Morozov, D. Jiang, M. I. Katsnelson, I. V. Grigorieva, S. V. Dubonos, and A. A. Firsov, Nature 438, 197 (2005)

[2] A. H. Castro Neto, F. Guinea, N. M. R. Peres, K. S. Novoselov, and A. K. Geim, Rev. Mod. Phys. 81, 109 (2009)

[3] L. Fu, C. L. Kane, and E. J. Mele, Phys. Rev. Lett.98, 106803 (2007);

[4] R. Roy, Phys. Rev. B79, 195322 (2009); J. Moore, Nature (London) 464, 194 (2010);

[5] M. Z. Hasan and C. L. Kane, Rev. Mod. Phys.82, 3045 (2010); X.-L. Qi and S.-C. Zhang, Rev. Mod. Phys.83, 1057 (2011).

[6] S. -Q. Sheng, Dirac Equation in Condensed Matter (Springer, Berlin, 2012); B. A. Bernevig and T. L. Hughes, Topological Insulators and Topological Superconductors (Princeton University Press, Princeton, 2013); Topological Insulators, Contemporary Concepts of Condensed Matter Science 6, eds. M. Franz and L. Molenkamp (Elsevier, Amsterdam, 2013).

[7] B. A. Bernevig, T. L. Hughes, and S. C. Zhang, Science 314, 1757 (2006).

[8] X. L. Qi, R. Li , F. Zang and S. C. Zhang, Science 323, 1184 (2009).

[9] Y. Zhang, Y.-W. Tan, H. L. Stormer, and P. Kim, Nature 438, 201 (2005).

[10] X. Huang, L. Zhao, Y. Long, P. Wang, D. Chen, Z. Yang, H. Liang, M. Xue, H. Weng, Z. Fang, X. Dai, and G. Chen, Phys. Rev. X 5, 031023 (2015).

[11] XY. Su et al, Science 349, 613 (2015).

[12] V. Pardo and W. E. Pickett, Phys. Rev. Lett. 102, 166803 (2009).

[13] S. Katayama, A. Kobayashi, and Y. Suzumura, J. Phys. Soc. Jpn. 75, 054705 (2006).
[14] P. Dietl, F. Piechon, and G. Montambaux, Phys. Rev. Lett. 100, 236405 (2008).

[15] Y. Wu, Opt. Express 22, 1906 (2014).

[16] P. Delplace and G. Montambaux, Phys. Rev. B 82, 035438 (2010).

[17] S. Banerjee, R. R. P. Singh, V. Pardo, and W. E. Pickett, Phys. Rev. Lett.103, 016402 (2009).

[18] M. Bahramy, B. -J. Yang, R. Arita, and N. Nagaosa, Nat. Commun. 3, 679 (2012).

[19] S. -Y. Xu, Y. Xia, L. A. Wray, S. Jia, F. Meier, J. H. Dil, J. Osterwalder, B. Slomski, A. Bansil, H. Lin, R. J. Cava, and M. Z. Hasan, Science 332, 560 (2011).

[20] J. Li, R.-L. Chu, J. K. Jain, and S. -Q. Shen, Phys. Rev. Lett.102, 136806 (2009); C. W. Groth, M. Wimmer, A. R. Akhmerov, J. Tworzydlo, and C. W. J. Beenakker, Phys. Rev. Lett.103, 196805 (2009); H.-M. Guo, G. Rosenberg, G. Refael, and M. Franz, Phys. Rev. Lett.105, 216601 (2010).

[21] I. Garate, Phys. Rev. Lett.110, 046402 (2013).

[22] O. Viyuela, A. Rivas, and M. A. Martin-Delgado, Phys. Rev. B 86, 155140 (2012); C.-E. Bardyn, M. A. Baranov, E. Rico, A. Imamoglu, P. Zoller, and S. Diehl, Phys. Rev. Lett. 109, 130402 (2012).

[23] Takashi Oka and Hideo Aoki, Phys. Rev. B79, 081406 (2009).

[24] N. H. Lindner, G. Refael, and V. Galitski, Nat. Phys, 7, 490 (2011).

[25] C.-K. Chan, P. A. Lee, K. S. Burch, J. H. Han, and Y. Ran, Phys. Rev. Lett.116, 026805 (2016).

[26] A. Gomez-Leon and G. Platero, Phys. Rev. Lett.110, 200403 (2013).

[27] Y. T. Katan and D. Podolsky, Phys. Rev. Lett.110, 016802 (2013).

[28] Y. H. Wang, H. Steinberg, P. Jarillo-Herrero, N. Gedik, Science 342, 453 (2013).

[29] J.-I. Inoue and A. Tanaka, Phys. Rev. Lett. 105, 017401 (2010).

[30] T. Kitagawa, E. Berg, M. Rudner, and E. Demler, Phys. Rev. B 82, 235114 (2010).

[31] P. M. Perez-Piskunow, G. Usaj, C.A. Balseiro, L.E.F. Foa Torres, Phys. Rev. B 89, 121401(R) (2014); P. M. Perez-Piskunow, L. E. F. Foa Torres, and G. Usaj, Phys. Rev. A91, 043625 (2015).

[32] A. G. Grushin, A. Gomez-Leon, and Titus Neupert, Phys. Rev. Lett.112, 156801 (2014).

[33] H. L. Calvo, L.E.F. Foa Torres, P.M. Perez-Piskunow, C.A. Balseiro, G. Usaj, Phys. Rev. B 91, 241404(R) (2015)

[34] J. Gonzalez and R. A. Molina, Phys. Rev. Lett. 116, 156803 (2016).

[35] R. Wang, B. Wang, R. Shen, L. Sheng, D. Y. Xing, Europhys. Lett. 105, 17004 (2014).

[36] A. Narayan, Phys. Rev. B 94, 041409(R) (2016); S. Ebihara, K. Fukushima, and T. Oka Phys. Rev. B 93, 155107 (2016); Z. Yan, Z. Wang, arXiv: 1605:04404.

[37] A. Narayan, Phys. Rev. B91, 205445 (2015).

[38] The Hamiltonian of a system with the usual 2D Dirac dispersion is $H_{0}(\mathbf{k})=v k_{x} \sigma_{x}+v k_{y} \sigma_{y}$. The perturbation, $m_{z} \sigma_{z}$ opens a gap and leads to the Chern number $\pm 1 / 2$ as a consequence of broken time-reversal symmetry $\left(\Theta=\sigma_{y} \mathcal{K}\right)$. Then the total Chern number is obtained by summing the contribution coming from all gapped Dirac nodes at the Fermi level within the BZ. The half semimetal without spin-orbit coupling may host 
such a dispersion [40]. Note that this model is little different from typical graphene where such a perturbation, namely, the "Semenoff mass" leads to zero Chern number since $C_{K}=-C_{K^{\prime}}$, where $K$ and $K^{\prime}$ are two Dirac points.

[39] H. Huang, Z. Liu, H. Zhang, W. Duan, and D. Vanderbilt, Phys. Rev. B92, 161115 (2015).

[40] Z. F. Wang, Zheng Liu, and Feng Liu, Phys. Rev. Lett.110, 196801 (2013).

[41] F. D. M. Haldane, Phys. Rev. Lett. 61, 2015 (1988).

[42] M. Bukova, L. D'Alessio, and A. Polkovnikov, Advances in Physics 64, 139 (2015).

[43] 2D organic metals or half-metallic systems may be promising candidates that can host only two Dirac nodes near the Fermi level.

[44] D. Culcer, A. MacDonald, and Q. Niu, Phys. Rev. B68,
045327 (2003).

[45] G. Montambaux, F. Piechon, J.-N. Fuchs, and M.O. Goerbig, Eur. Phys. J. B 72, 509 (2009)

[46] The Berry curvature in a gapped SD system turns out to be $\frac{v}{2\left|n_{\mathrm{g}}(\mathbf{k})\right|^{3}}\left[\gamma\left(\alpha k_{x}^{2}-\delta_{1}\right)-\eta k_{y}\left(\alpha k_{x}^{2}+\delta_{1}\right)+m \xi\left(k_{y} \eta+\gamma\right)\right.$ $\left.+2 m k_{x}\left(\alpha+\beta \xi-\eta \chi\left(\alpha k_{x}^{2}-\delta_{1}\right) / v\right)-m^{2} k_{x} \eta \xi \chi / v\right]$.

[47] L. E. F. Foa Torres, P. M. Perez-Piskunow, C. A. Balseiro, G. Usaj, Phys. Rev. Lett.113, 266801 (2014).

[48] H. Dehghani, T. Oka, and A. Mitra, Phys. Rev. B91, $155422(2015)$

[49] J. Karch, P. Olbrich, M. Schmalzbauer, C. Zoth, C. Brinsteiner, M. Fehrenbacher, U. Wurstbauer, M. M. Glazov, S. A. Tarasenko, E. L. Ivchenko, D. Weiss, J. Eroms, R. Yakimova, S. Lara-Avila, S. Kubatkin, and S. D. Ganichev, Phys. Rev. Lett.105, 227402 (2010). 